\title{
Chinese guideline on sublingual immunotherapy for allergic rhinitis and asthma
}

Huabin $\mathrm{Li}^{1 \#}$, Shi Chen ${ }^{2 \#}$, Lei Cheng ${ }^{3,4 \#}$, Yinshi Guo ${ }^{5 \#}$, He Lai ${ }^{6 \#}$, Yong Li $^{\text {7\#}}$, Xiaoping Lin ${ }^{8 \#}$, Zheng Liu", Qianhui Qiu ${ }^{10 \#}, J_{i e}$ Shao ${ }^{11 \#}$, Li Shi $^{12 \#}$, Man Tian ${ }^{13 \#}$, Chengshuo Wang ${ }^{14 \#}$, Hongtian Wang ${ }^{15,16 \#}$, Xueyan Wang ${ }^{16 \#}$, Qingyu Wei ${ }^{8 \#}$, Yongxiang Wei ${ }^{17 \#}$, Li Xiang $^{18 \#}$, Qintai Yang ${ }^{19 \#}$, Changqing Zhao ${ }^{20 \#}$, Huanping Zhang ${ }^{21 \#,}$ Yuxiang Zhi ${ }^{22 \#}$, Junxiao Gao ${ }^{10}$, Quansheng Li $^{8}$, Juan Liu' ${ }^{1}$, Kuiji Wang ${ }^{14}$, Wencheng Zhou ${ }^{3,4}$, Luo Zhang ${ }^{14}$; Chinese Society of Allergy (CSA)

${ }^{1}$ Department of Otolaryngology, Affiliated Eye, Ear, Nose and Throat Hospital, Fudan University, Shanghai 200031, China; ${ }^{2}$ Center for Prevention and Treatment of Pediatric Asthma, Hainan General Hospital, Haikou 570102, China; ${ }^{3}$ Department of Otorhinolaryngology, The First Affiliated Hospital, Nanjing Medical University, Nanjing 210029, China; ${ }^{4}$ International Centre for Allergy Research, Nanjing Medical University, Nanjing 210029, China; ${ }^{5}$ Department of Allergy \& Immunology, Renji Hospital, School of Medicine, Shanghai Jiaotong University, Shanghai 200127, China; ${ }^{6}$ Department of Allergy, The Second Affiliated Hospital, Guangzhou Medical University, Guangzhou 510260, China; ${ }^{7}$ Department of Otolaryngology, Head and Neck Surgery, Affiliated Hangzhou First People's Hospital, Zhejiang University, Hangzhou 310006, China; ${ }^{8}$ Department of Allergy, General Hospital of Northern Theater Command, Shenyang 110016, China; ${ }^{9}$ Department of Otolaryngology-Head and Neck Surgery, Tongji Hospital, Tongji Medical College, Huazhong University of Science and Technology, Wuhan 430030, China; ${ }^{10}$ Department of Otolaryngology, Zhujiang Hospital, Southern Medical University, Guangzhou 510282, China; ${ }^{11}$ Department of Pediatrics, Ruijin Hospital Affiliated to Shanghai Jiaotong University School of Medicine, Shanghai 200025, China; ${ }^{12}$ Department of Otolaryngology-Head and Neck Surgery, Shandong Provincial ENT Hospital, Shandong University, Jinan 250022, China; ${ }^{13}$ Respiratory Department, Children's Hospital of Nanjing Medical University, Nanjing 210008, China; ${ }^{14}$ Department of Otolaryngology Head and Neck Surgery, Beijing Tongren Hospital, Capital Medical University, Beijing 100073, China; ${ }^{15}$ Department of Otolaryngology, Head and Neck Surgery, The First Medical Center, Chinese PLA General Hospital, Beijing 100853, China; ${ }^{16}$ Department of Allergy, Beijing Shijitan Hospital, Capital Medical University, Beijing 100038, China; ${ }^{17}$ Department of Otolaryngology, Beijing Anzhen Hospital, Capital Medical University, Beijing 100029, China; ${ }^{18}$ Department of Allergy, Beijing Children's Hospital, Capital Medical University, National Center for Children's Health, Beijing 100045, China; ${ }^{19}$ Department of Otorhinolaryngology-Head and Neck Surgery, The Third Affiliated Hospital, Sun Yat-sen University, Guangzhou 510630, China; ${ }^{20}$ Department of Otolaryngology, Second Hospital of Shanxi Medical University, Taiyuan 030001, China; ${ }^{21}$ Department of Pulmonary and Critical Care Medicine, First Hospital of Shanxi Medical University, Taiyuan 030001, China; ${ }^{22}$ Department of Allergy, Peking Union Medical College Hospital, Beijing 100730, China

\#These authors contributed equally to this work.

Correspondence to: Luo Zhang, MD, PhD. Department of Otolaryngology Head and Neck Surgery, Beijing Tongren Hospital, Capital Medical University. No. 1, Dongjiaominxiang, Dongcheng District, Beijing 100073, China. Email: dr.luozhang@139.com.

Submitted Oct 24, 2019. Accepted for publication Dec 01, 2019.

doi: $10.21037 /$ jtd.2019.12.37

View this article at: http://dx.doi.org/10.21037/jtd.2019.12.37

\section{Introduction}

The present guideline on sublingual immunotherapy (SLIT) for allergic rhinitis (AR) and allergic asthma (AS) has been developed by a panel of experts on behalf of the Chinese Society of Allergy to guide the clinical practice of SLIT in China. The panel included eminent clinical physicians and scientific specialists, who majored in the fields of Otolaryngology, Respirology, Pediatrics, Allergy and Clinical Immunology, and focused their investigations on two common allergic airway diseases (namely AR and AS) in China. During the past few decades, AR and AS have shown increasing prevalence worldwide, and resulted in substantial direct and indirect costs (1). Recent epidemiological studies have also indicated a high prevalence of AR and AS in China (2-5). Both AR and AS can cause sleep impairment and reduce school and work performance, resulting in significant social and economic burdens (1). The current treatment options for AR and AS 
include allergen avoidance, pharmacotherapy, allergenspecific immunotherapy (AIT) and patient education to preferably guide clinical practice for all practitioners (6). Unlike allergen avoidance and pharmacotherapy, which offer temporary symptom-relief, AIT is the only option that is disease-modifying and may alter the natural course of allergic response and provide symptomatic relief after discontinuation of therapy (6). AIT has been used in clinics for more than one hundred years, and its efficacy and safety have been well confirmed as both subcutaneous immunotherapy (SCIT) and SLIT (7). Similar to SCIT, SLIT can exert long-term relief of nasal and bronchial symptoms, as well as prevent new sensitization and development of AS. Moreover, SLIT with a single allergen vaccine can achieve good efficacy in polysensitized patients with AR and AS (8). Notably, SLIT has now been used for over a decade with standardized house dust mite (HDM) regimens in China. Indeed, a series of published clinical trials have demonstrated the efficacy of SLIT in HDM-induced AR and AS in children and adults, and thus based on these clinical trials, this SLIT guideline is herein organized to outline the critical items, including the epidemiology of AR and AS, molecular mechanisms, indications and contraindications of SLIT, standardized allergen preparation, efficacy evaluation and management of adverse events (AEs) of SLIT; to guide and improve the efficacy and safety of SLIT in clinical practice.

\section{Epidemiology and sensitized allergens of AR and AS in China}

$\mathrm{AR}$ is one of the most common allergic diseases with high incidence and prevalence affecting over $10 \%$ to $40 \%$ of the population worldwide (9). In China, similar incidence with increased tendency has been observed in the past few years. A population-based national study surveyed over 38,000 adult subjects in 11 major cities across China from September 2004 to May 2005 using validated questionnaire-based telephone interviews, and showed that the self-reported prevalence of AR was 11.1\% (8.7-24.1\%) (10). A follow-on survey involving a total of 47,216 telephone interviews in adults in 18 major cities across China after 6 years indicated that the standardized AR prevalence has significantly increased to $17.6 \%(9.8-23 \%)(11)$. It is noteworthy that the grasslands of northern China show a high prevalence of pollen-induced AR. A study involving 6,043 subjects in the grasslands of northern China undergoing face-toface interviews and skin prick test (SPT) from December
2009 to March 2010, has shown a prevalence of $32.4 \%$ epidemiologic AR and $18.5 \%$ physician-diagnosed polleninduced AR (12).

Increased tendency in prevalence has been similarly observed in children in China as in other countries (13). In this regard, it is estimated that the prevalence of selfreported $\mathrm{AR}$ among children worldwide is about $2 \%$ to $25 \%$, with a $>20$-fold variation among countries in the same region (14). In China, a series of studies performed in different areas have reported AR prevalence rate of around $15 \%$. For example, in 2005, a questionnaire survey combined with SPT performed in children aged 3 to 6 years in Wuhan of China, indicated the prevalence of AR to be $10.8 \%$ (15). Similarly, a cross-sectional survey of children aged $0-14$ years in Beijing, Chongqing, and Guangzhou in 2008-2009 has shown the self-reported AR prevalence rates to be $14.46 \%, 20.42 \%$, and $7.83 \%$, respectively (4). Another study investigating the prevalence of AR among elementary and middle school students in Changsha, from June 2011 to April 2012, reported the prevalence of AR to be between $15.8-19.4 \%$ (16). Similar to findings from the westernizedcountries, the AR prevalence in China has also been shown to be different in developed and undeveloped areas. For example, a survey of children aged 3 to 5 years has revealed that the prevalence of $\mathrm{AR}$ in urban and suburban areas of Beijing was $19.5 \%$ and $10.8 \%$, respectively (17). A more recent study from Shanghai investigating AR subclassification in children has indicated that $8.6 \%$ of children had intermittent mild AR, $4.2 \%$ persistent mild AR, $40.5 \%$ intermittent moderate-severe AR and $46.7 \%$ persistent moderate-severe AR (18).

Asthma is the most common co-morbidity of AR, which affects children and adults of all ages (19). Epidemiologic data obtained from the CARE (China Asthma and Risk factors Epidemiologic) study conducted from February 2010 to August 2012, indicated that there were about 30 million asthma patients in China during this period (20). Furthermore, the result demonstrated that the prevalence of asthma among individuals aged $>14$ years was $1.24 \%$, with no gender difference, and had increased over the past 10 years. Similarly, the latest nation-wide cross-sectional survey of nearly 500,000 children aged $0-14$ years enrolled from urban areas in 43 cities across China using a cluster-stratified sampling method, between September 2009 to August 2010, has indicated that asthma has also increased in this age group (5). The survey demonstrated that total childhood asthma incidence increased from $1.09 \%$ in 1990 , to $1.97 \%$ in 2000 , and $3.02 \%$ in 2010 . 
Moreover, the highest prevalence of childhood asthma was in eastern China (4.23\%) and the lowest in northeast China (2.00\%) (5).

The prevalence rate of inhaled allergens sensitization varies widely among regions. Dermatophagoides farinae (Der $f$ ) and Dermatophagoides pteronyssinus (Der p) are shown to be the major allergens, which induce AR and/ or AS in China. The regional distribution patterns of both $\operatorname{Der} f$ and $\operatorname{Der} p$ sensitization are the highest in the southern and central parts of China, and the lowest in the northern regions, especially in the northwest (21). A cross-sectional survey of 6,304 patients with AS and/or $\mathrm{AR}$ in 17 cities from 4 regions of China, tested for SPT positivity to 13 common aeroallergens has shown that the overall sensitization was highest to $\operatorname{Der} f(59.0 \%)$ and $\operatorname{Der} p(57.6 \%)$ (22). Moreover, according to a large crosssectional multicenter epidemiological study from China, $83.7 \%$ of the participants were concomitantly sensitized to $\operatorname{Der} f$ and $\operatorname{Der} p(21)$. However, airborne pollen is the most frequent and seasonal cause of allergy in the western and northern China. In northern China, a multistage, clustered and proportionately stratified random sampling with a fieldinterviewer administrated SPT survey of 6,043 patients has shown that Artemisia, chenopodium and bumulus scandens were the most common sensitizing pollen types in $18.5 \%$ patients with $\mathrm{AR}$ (12).

\section{Clinical practice of SLIT in AR}

Historically, SCIT with non-standardized allergen regimen has been performed since the 1950s in China (23). The concept of standardized allergen vaccine was formally proposed in 1983 (24), while the first SLIT product "Chanllergen", a vaccine made with a single extract of $\operatorname{Der} f$, was approved by China Food and Drug Administration (CFDA) in 2006 for the clinical treatment of AR and AS patients (23). Subsequently, the first studies addressing SLIT efficacy and safety in children and adults with AR in China were published in 2007 and 2011, respectively $(25,26)$. Thus, as a disease-modifying treatment option with well documented safety and tolerability, SLIT is currently recommended as a first-line treatment for AR in China $(13,27)$.

In China, SLIT has been shown to be effective, with short-term and long-term clinical benefits, in AR patients by several clinical trials. Different courses of SLIT have been shown to significantly decrease the total nasal symptom score (TNSS), total medications score (TMS), visual analogue scale (VAS), and/or rhinoconjunctivitis quality of life questionnaire (RQLQ) score in both children and adult patients with AR caused by HDM (26,28-36) (Table 1). While, there is some evidence of a certain rebound in the nasal symptoms one year after the end of 2-year SLIT (34), a 3 -year course of SLIT has been found to be more effective in achieving a higher proportion of medication withdrawal than 1- or 2-year course (31), indicating that a 3-year SLIT might be more suitable for AR patients. Furthermore, it has been demonstrated that despite more than $90 \%$ of individuals being sensitized to two or more allergens (22), SLIT with single-allergen is effective in both monosensitized and polysensitized children and adults with AR; with equivalent efficacy achieved after 1.5-2 years (30). Indeed, SLIT has been shown to be a particularly effective treatment in AR children. A randomized placebo control study of SLIT with $\operatorname{Der} f$ drops in 30 children demonstrated that the symptom scores were significantly decreased after 1 and 2 years of SLIT compared to the placebo group; with the symptom score significantly decreased after 2 years of SLIT compared with 1 year of SLIT (47). Other studies involving 2 years of SLIT with $\operatorname{Der} f$ drops have also shown significant improvements in TNSS, TMS and VAS $(48,50)$. One recent study has demonstrated that SLIT combined with standard therapy was also significantly more effective than standard therapy in attaining and maintain asthma control in pediatric patients after 6 months $(75.6 \%$ vs. $60.3 \%)$ and 2 years $(89.7 \%$ vs. $66.7 \%$ ) following SLIT (44). Furthermore, one more recent prospective long-term study has demonstrated that the benefits of SLIT could last for up to 7 years after a 2-year SLIT in monosensitized and polysensitized AR children; although monosensitized children generally achieved more sustained benefit than polysensitized children (49).

SLIT is a safer alternative to traditional SCIT, as it elicits fewer and milder AEs; including both local and systemic AEs. The local AEs of SLIT mainly include sublingual itching or redness; however, gastrointestinal reactions such as abdominal pain and diarrhea may occur when the allergen is swallowed. Most AEs usually occur in the first week of SLIT; with only $0.056 \%$ of allergic patients experiencing systemic reactions, and less than 1.4 serious AEs (including asthma attacks, abdominal pain, vomiting, staphyledema and urticaria, etc.) occurring per 100,000 sublingual administrations (8). Several reports have summarized the AEs of SLIT in Chinese AR patients during the past decade, and indicated that the incidence of AEs in these patients 
ranges from $8.4 \%$ to $27.7 \%(33,38,40,43)$. However, most AEs were reported to be slight local reactions and resolved within a few days without any medical intervention, whereas other AEs were resolved after dose adjustments suggested by the physician. Currently, no serious AEs of SLIT have been reported in China.

The molecular mechanisms underlying AIT efficacy have not been fully clarified. AIT was first proposed by British medical experts Noon and Freeman around 1911 (51), who realized that AR (hay fever) was caused by inhalation of subtilis toxin (an allergen found to be present in hay), and subcutaneous injection of subtilis toxin into AR patients achieved significant clinical benefits. However, the key mechanism of action of SCIT was found to be not its socalled antitoxin effect, but immune tolerance after repeated injection of allergen. Recent advances have revealed that SCIT is critical in regulating a variety of immune cells; including regulatory $\mathrm{T}$ cells (Tregs), B cells, and dendritic cells; for modification of the immune system, resulting in the tolerance to allergens. Similar to SCIT, SLIT also orchestrates multiple pathways in modulating the immune system. There is some evidence that the local dendritic cells in the oral mucosal immune system capture the sublingual allergens and initiate the $T$ cell response that triggers the sensitization mechanism (52). Indeed, numerous studies by Chinese investigators have shown that $\mathrm{T}$ cell and B cell responses are significantly altered upon SLIT administration in AR patients. Importantly, Th2-dominant response was found to be continuously attenuated and replaced by a Th1-driven immune response upon SLIT administration, as manifested by decreased release of IL4, IL-5, and IL-13 from PBMCs-derived Th2 cells (43,53). One study has revealed significant TIM-1 suppression with efficient SLIT in HDM-induced AR patients (38). Some studies have also indicated the involvement of microRNA related immune modification in SLIT. For example, expression of mir-146a, a critical micro-RNA inhibiting Th2 immune response, was shown to be increased in children with AR after SLIT (45). Moreover, after SLIT administration, Th2 immune response was downregulated through TSLP-OX40L signaling pathway in patients with persistent moderate to severe AR (32). SLIT has also been shown to induce Tregs with increased IL-10 levels (54) and in SLIT with HDM regimen, clinical efficacy was achieved through TGF- $\beta$ mediated immunological suppression and an increase in Tregs (55). Furthermore, synthesis of antigen-specific immunoglobulin $\mathrm{E}$ (IgE) has been shown to be inhibited and production of immunoglobulin G4 (IgG4) blocking antibody increased after SLIT administration (56). Data from one multicenter, random, open control study has also indicated that serum specific IgG4 antibody levels are increased significantly upon SLIT administration compared with the control group, while the ratio of $\mathrm{IgE} / \mathrm{IgG} 4$ declines compared to the baseline (40).

\section{Clinical practice of SLIT in AS}

SLIT has also been used for AS patients in China for over ten years; with the first SLIT clinical trial in AS children published in 2007 (25). Although the effect of SLIT for AS patients has been documented by several studies $(37,46)$ (Table 1), it has not yet been a common treatment choice in adults with AS. A retrospective study conducted in 134 adult patients with HDM-induced AS showed that 2 years SLIT with pharmacotherapy was more effective than conventional drug treatment, as indicated by significantly lower clinical scores; including daytime/nighttime asthma symptom score, medicine score, improved peak expiratory flow (PEF) rate, asthma control test and asthma control questionnaire (ACQ) score (46). Another open-label trial with $\operatorname{Der} f$ drops also appeared to significantly decrease the bronchial symptom score after 1-year treatment (37).

SLIT has been shown to be effective in both pediatric and adult AS patients by attenuating the bronchial symptoms and improving the respiratory functions, as well as lowering the dosages of inhaled glucocorticoid and $\beta 2$ agonists. However, there are more studies of SLIT in children than in adults with AS (8). Two early randomized double-blind placebo control (RDBPC) trials have demonstrated that $\operatorname{Der} f$ drops significantly improved the symptoms and reduced drug use over the entire course of treatment in Chinese asthmatic children $(25,39)$. One of these trials, involved 278 AS patients aged 4-18, and showed that half-a-year SLIT with $\operatorname{Der} f$ drops significantly improved the lung function with the selfreported efficacy of $68.5 \%$ in the active treatment group compared to $16.4 \%$ in the placebo group (25). Another RDBPC trial involving 112 AS children aged 4-13 years showed similar clinical benefits in both monosensitized and polysensitized children after one year of SLIT (41). A recent study has compared the effect of different course of SLIT in children with AR and AS and demonstrated that the improvement in symptoms and particularly VAS score after 3 years of SLIT was significantly greater than improvements after 1 or 2 years' SLIT, but not after 4 years' SLIT, suggesting that a 3 -year treatment course 


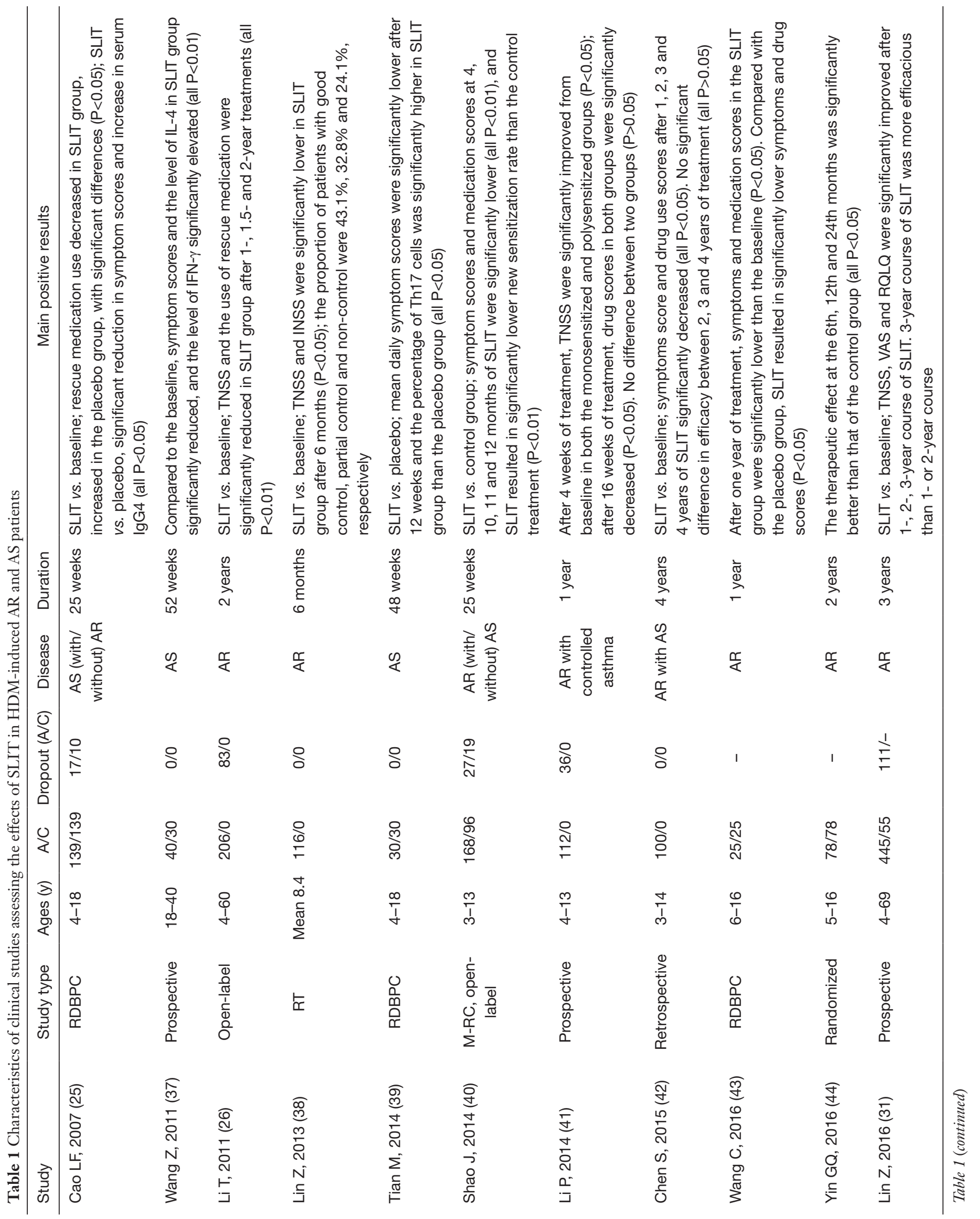




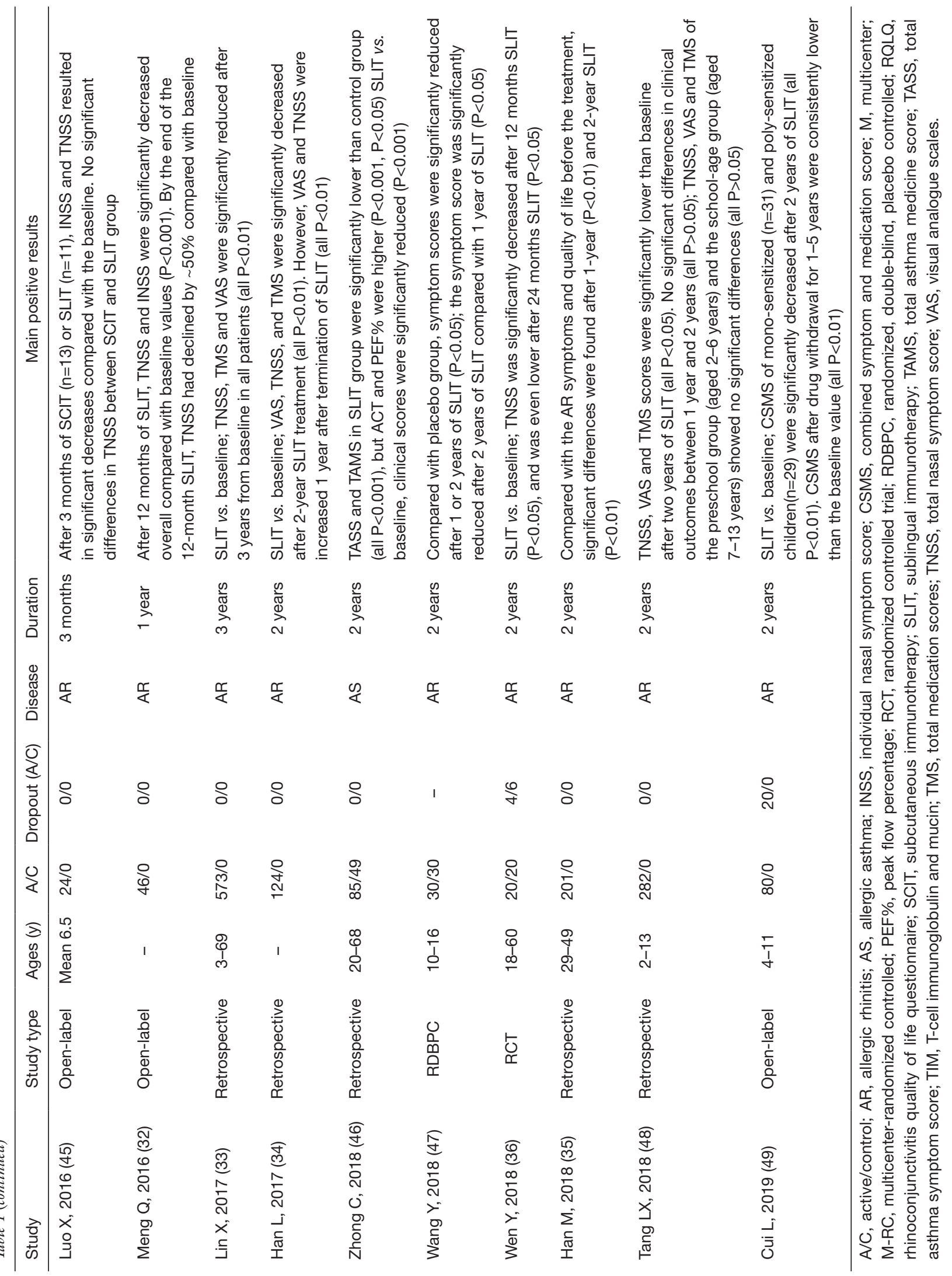


with SLIT is optimal (42).

Comparable safety of SLIT has been demonstrated in both AR and AS patients; however, SLIT is not recommended in patients with severe persistent and uncontrolled asthma. Thus, prior to initiation of SLIT, standardized questionnaires should be performed to verify the condition of asthma control, including the asthma control test (ACT) and spirometry or peak flow measurement before and after dosing.

The AEs reported in most SLIT studies involving AS patients in China were local, mild or moderate, without severe anaphylaxis, and found to occur in $0 \%$ to $20 \%$ of the patients $(31,40,41,44,46)$. The local AEs were mainly oral or sublingual itching, swelling, and diarrhea (27). The incidence of AEs varied widely, and most AEs occurred in the first two weeks of treatment $(8,57,58)$.

Tolerogenic immune responses similar to those in AR patients are also observed in AS patients after SLIT. In particular, there is a shift from Th2 (secreting proinflammatory cytokine: IL-4, IL-5, IL-13, etc.) to Th1 (secreting anti-inflammatory cytokine: TGF- $\beta$, IL-10, IL-35, etc.) response $(43,59)$. Moreover, Tregs are also induced. For example, the numbers of cluster of differentiation CD4+CD25+Treg cells have been shown to be significantly increased during SLIT with $\operatorname{Der} f$ drops in patients with HDM-induced AS (39), and specific IgG4 distinctly increased after SLIT for 12 months (40).

\section{The standardized procedure of SLIT}

The standardized procedure of SLIT mainly includes the diagnosis of allergic diseases, standardized prescription, initial clinical education for patients in hospital, initial drug use, establishment of patient files and regular followup education until the whole course of desensitization is completed (Figure 1).

\section{Medical staff training}

Professional knowledge training regarding SLIT should be conducted regularly for medical staff in desensitization centers. Medical staffs need to carry out allergen sensitization tests (SPT and/or serum sIgE tests) in patients suspected to have AR and/or AS, and assess whether positive allergen test results are consistent with allergic history. Although SLIT is much safer than SCIT, the occurrence of severe AEs of SLIT need to be recognized, and first aid given immediately as appropriate. Regular identification, treatment and first aid exercises on the common adverse reactions of SLIT should also be conducted. In order to evaluate and improve patient compliance and efficacy, implement public education plan, and reduce the incidence of adverse reactions, patient files should be established and follow-up should be carried out at three-month intervals.

\section{Establishment of patient files}

Patient symptoms, signs, medical history, allergen test results, other related auxiliary examination results, the first prescription of SLIT, and anti-allergic and other drug usage must be recorded to establish patient files. Medical staff should also determine and agree the time of re-visit and follow-up arrangements with patients.

\section{Prescription of standardized SLIT}

If a patient meets the diagnostic criteria of allergic disease and is willing to receive SLIT, physicians can prescribe standardized SLIT and anti-allergic drugs according to the patient's symptoms. After the patient gets the SLIT preparation from the pharmacy with a prescription, he/ she should return to the designated science education area of the consulting rooms to receive the initial clinical education and the first dose of SLIT under the supervision of the physician. The patient should be observed for at least 30 minutes after administration of the SLIT dose before being discharged (60).

\section{Follow-up and patient education}

The purpose of follow-up and patient education is to constantly evaluate the efficacy and adverse reactions, as well as continuously improve compliance. It has been reported that $54 \%$ of the patients drop-out within the first year of SLIT; mainly because patients could not be reached, ineffectiveness of treatment, and inability to adhere to long-term treatment (61). Moreover, most drop-outs occur in the first two months of the initial treatment (62). Clinical visits are recommended to be conducted at least at three monthly intervals because patients followed up once or four times a year during SLIT have demonstrated total drop-out rates of $41.1 \%$ or $10.4 \%$, respectively, two years later (63). Thus, it can be concluded that especially the first two months in the first year of SLIT are the key period to prevent drop-outs and regular follow-up may significantly improve patient compliance. 


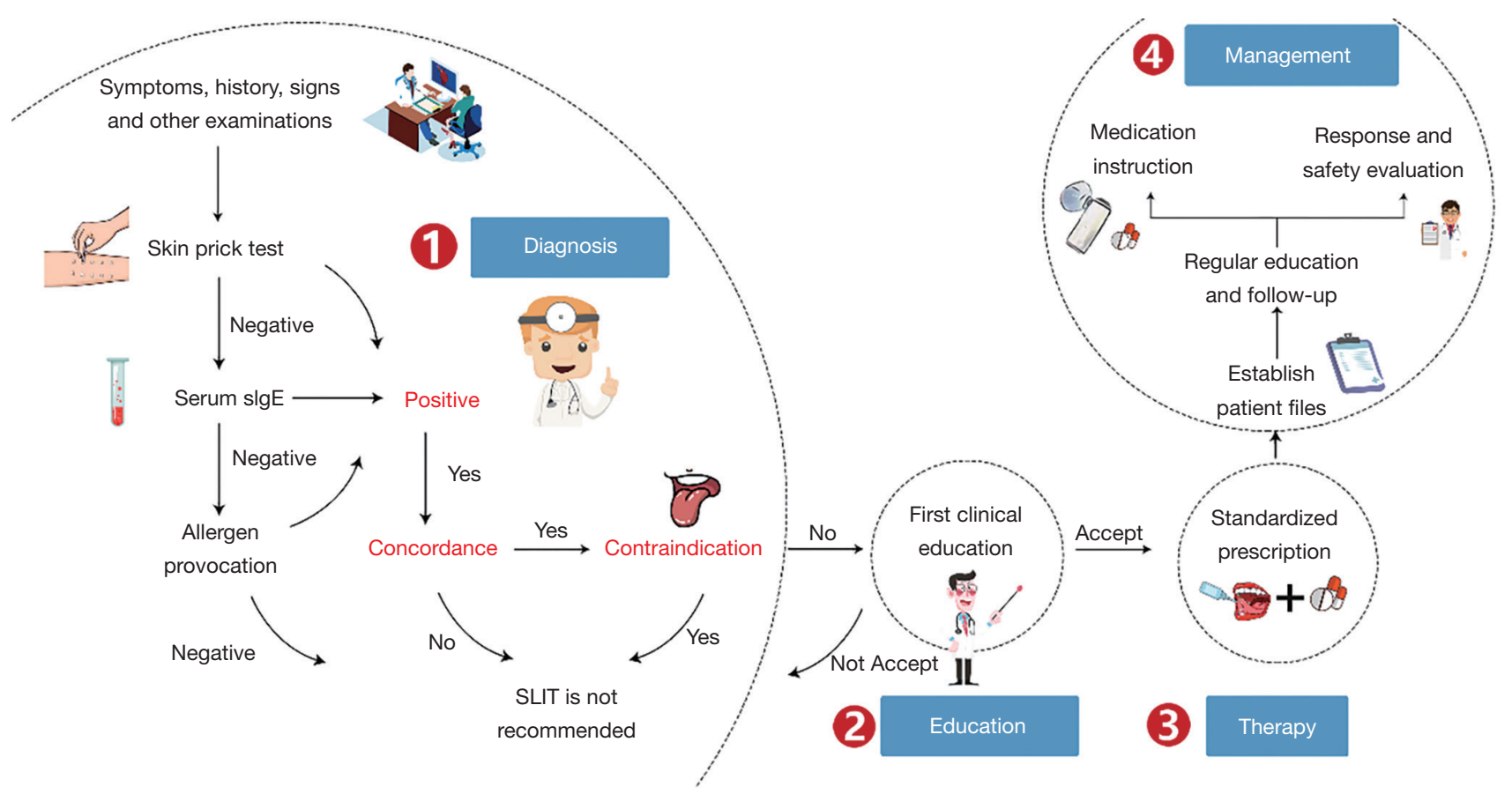

Figure 1 A schematic view of the concise process for standardized SLIT. SLIT, sublingual immunotherapy.

\section{Initial clinical and follow-up education and others}

The initial clinical education session involves giving patients information on the following items: (I) the nature, causes and hazards of AR and AS; (II) the characteristics of SLIT and its relationship with anti-allergic drugs; (III) the methods, courses of treatment, costs, efficacy and safety of SLIT; (IV) the need to obtain face to face informed consent from children and adults receiving SLIT treatment, while the guardians of young children or high-sensitivity children need to sign the written consent; and (V) advice over telephone or written guidance on how to avoid allergen and deal with adverse reactions.

Follow-up visits are supplement and continuation of the initial clinical education, and an important measure to increase compliance. Follow-up education includes: (I) obtaining information on the patient's recent clinical symptoms, signs and drug use; (II) adjusting the SLIT program through efficacy evaluation; (III) confirming incidence of any adverse reactions, guiding patients on how to identify the warning signs of adverse reactions and the corresponding treatment methods, as well as how to seek medical treatment in time; (IV) instructing patients how to avoid inhalation of allergens in the environment. Each follow-up should be recorded in the patient files in detail to provide the basis for the evaluation of curative effect and the adjustment of the plan.

\section{Family education}

Management of family education is indispensable in the comprehensive education program, as it guarantees continuous standardization of immunotherapy. Physicians can popularize the knowledge of mite allergy to patients and their families at the initial clinical education, by telephone follow-up and regular health-related lectures, as well as guide family members to supervise the patient's standardized medication (64).

\section{Popular science education}

Popular science education includes the following items: (I) publicity brochures and wall-mounted information sheets related to allergic diseases should be placed in outpatient clinics; (II) medical consulting services such as telephone, short message and WeChat groups should be available for patients; (III) desensitization centres should be established, lectures and knowledge competitions should be held regularly through publicity and advertisements in newspapers and magazines, as well as free clinics for NoseLoving Day, World Asthma Day and World Allergy Day. 


\section{Storage of standardized SLIT drugs}

SLIT preparations should be stored in a dark, airtight and cool container (no more than $20^{\circ} \mathrm{C}$ ), away from the reach of children.

\section{Indications and contraindications of SLIT in AR and AS patients}

\section{Indications}

AIT is highly recommended for AR and AS patients, if the sensitizing allergens are clearly identified by SPT and/or serum sIgE tests, and confirmed to be the cause of the disease (13). SLIT can be used as an initial and early treatment strategy for respiratory allergic diseases, especially for the following patients (8): (I) patients whose symptoms cannot be effectively controlled by conventional anti-allergic drugs; (II) patients who experience unacceptable adverse reactions with the anti-allergic drug treatment; (III) patients who refuse to be treated by injection; and (IV) patients who worry about side effects of long-term drug treatment or do not adhere to long-term drug treatment. At present, Der $f$ drop preparation is the only standardized SLIT agent available for clinical use in China, and indicated mainly for AR and AS caused by dust mite allergen, with/without some other allergens.

\section{Contraindications}

The contraindications of SLIT can be either absolute or relative. Absolute contraindications are as follows: (I) severe or uncontrolled asthma (forced expiratory volume in the first second, FEV $1<70 \%$ predicted value) and irreversible respiratory obstructive diseases; (II) patients with active malignant neoplasias; (III) patients with acquired immune deficiency syndrome (AIDS); (IV) children under 2 years of age; and $(\mathrm{V})$ pregnancy (do not start a new AIT). Similarly, relative contraindications are as follows: (I) partially controlled asthma; (II) patients being treated with $\beta$-blockers (systemic or local) or angiotensinconverting enzyme (ACE) blockers; (III) severe cardiovascular diseases; (IV) human immunodeficiency virus (HIV) infection; (V) immunodeficiency; and (VI) patients with severe psychological disorders or unable to understand the risks and limitations of the treatment. Doctors should consider the "risk versus benefit" balance when prescribing SLIT, based on an evaluation of the patient's medical condition (65).

\section{Therapeutic process}

\section{Conventional therapeutic process of SLIT with HDM extract}

The biologically standardized Der $f$ extracts should be prepared as five stock solutions, labelled No. 1 to No. 5 with increasing concentrations of total protein $(1,10,100,333$ and $1,000 \mu \mathrm{g} / \mathrm{mL}$ ), and used in the form of drops. As SLIT allows self-administration at home, without the supervision of a physician, the patient should be instructed to administer the prescribed drops under the tongue for 1-3 minutes before swallowing, at a certain time of the day. Oral intake is allowed 15 minutes after each treatment. In routine use, the treatment consists of an up-dosing phase and a maintenance phase; with a treatment course of 3 years recommended to achieve long-term efficacy (66). The specific daily dosing schedules of Der $f$ drops are shown in Table 2 (31).

\section{Individualized treatment}

In most cases of clinical application, SLIT allows selfadministration at home. However, individual differences in the effectiveness and side-effect profiles should be considered, and the doses should be adjusted accordingly to suit individual cases. Thus, the follow-up is of vital importance in the process of SLIT. Also, it is crucial to use a clinically effective dose from the onset of treatment (67).

\section{Measurements of efficacy}

SLIT efficacy should be evaluated in terms of patient selfassessment and physician-assessment, in terms of symptom score, quality of life questionnaire and measurement of medication score $(60,68,69)$. Objective measures can also provide impersonal proof to assess the effectiveness of immunotherapy (69). As biomarkers for predicting the efficacy of SLIT remains controversial (23), it is advisable to evaluate the efficacy at every three monthly interval during continuous treatment with SLIT.

\section{Measures of the efficacy of SLIT in AR patients}

\section{Nasal symptoms score}

A 4-point scale (from $0=$ none to $3=$ severe) is a method commonly used to rate the severity of AR symptoms in SLIT clinical trials (69). The method assesses the severity of each of four specific nasal symptoms; including nasal congestion, sneezing, nasal itching, and nasal secretion; 
Table 2 SLIT schedule with $\operatorname{Der} f$ drops in AR patients

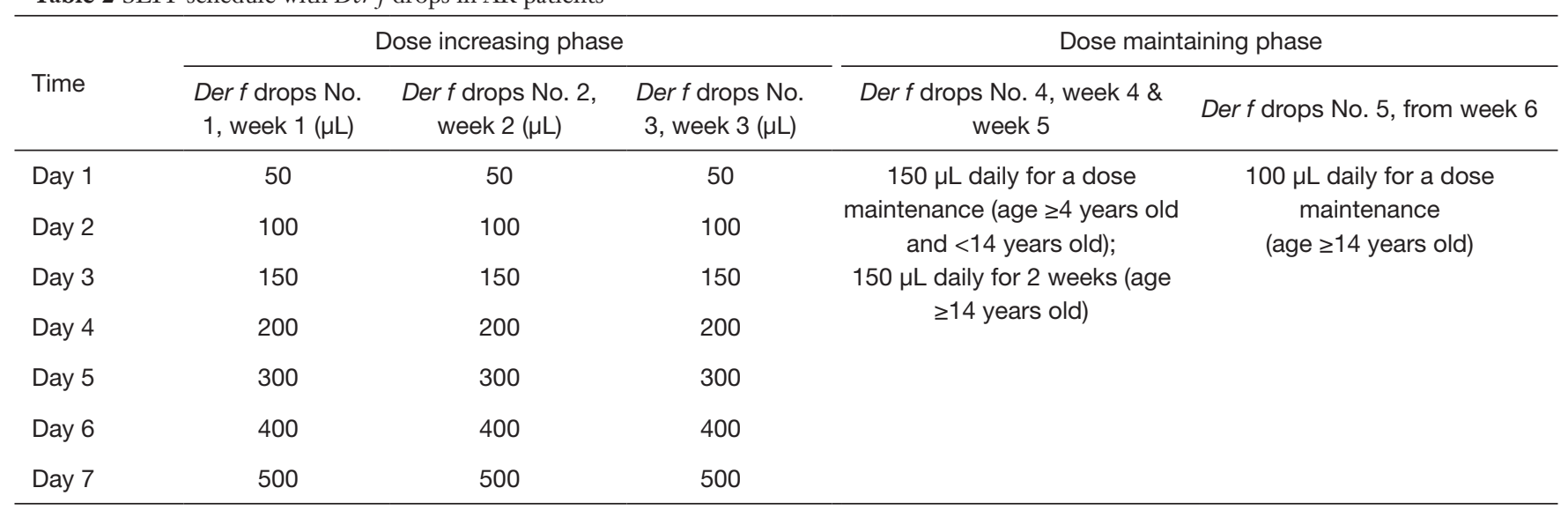

SLIT, sublingual immunotherapy; AR, allergic rhinitis; Der $f$, Dermatophagoides farinae.

from 0 to 3 , to provide the total nasal symptoms score (TNSS) ranging from 0 to 12 (60). Ocular symptoms such as itching, secretion and redness, are also each scored similarly from $0-3$ points, to provide the total ocular symptoms score (TOSS) ranging from 0 to 9 points. Alternatively, a VAS of 0 to 10 points (with $0=$ no symptoms and $10=$ very severe symptoms) has also been employed in some studies for assessing the subjective symptoms of patients. The VAS is a good measure for assessing the severity of the patient's disease, and is recommended to evaluate the subjective symptoms of patients on a weekly basis (60).

\section{Quality of life questionnaire}

In order to assess the "overall" efficacy of SLIT, a patient's mental/psychological state and health-related quality of life also need to be evaluated in addition to the effect on nasal symptoms. In this regard, the RQLQ, a disease-specific quality of life questionnaire, is most commonly scoring system employed in investigations of SLIT (70).

\section{Medication score}

Not all patients are completely symptom-free during the treatment of SLIT, and clinicians may give appropriate medications to relieve AR symptoms. In this regard, the effect of SLIT is assessed on the TMS, which is derived according to the use of traditional pharmacological agents scored based on the WAO guidelines as follows: antihistamines for nasal, ocular or oral use $=1$ point; corticosteroids for inhalation or nasal use $=2$ points; and corticosteroids for oral use $=3$ points $(60,71)$.

\section{Objective measures}

The symptoms and medication scores cannot be replaced by functional measurements. Nevertheless, the function measures can provide objective supporting evidence for the efficacy of SLIT. Suitable objective functional measurements include nasal airway resistance (NAR), acoustic rhinometry and nasal mucosa provocation test $(69,72)$.

\section{Measures of the efficacy of SLIT in AS patients}

\section{Asthma daytime/nighttime symptom scores}

Effect of SLIT on asthma symptoms (cough, chest tightness, shortness of breath, and wheezing) is commonly scored according to a daytime symptom score based on a scale of $0-5$ points, and a nighttime symptom score based on a scale of $0-4$ points, according to both the severity of symptoms and their impact on life (Table 3). The sum of daytime and nighttime asthma symptom scores provides the total asthma symptom score (TASS) (25).

\section{Total asthma medicine score (TAMS)}

Rescue medicine use in asthma patients needs to be assessed during SLIT. Asthma patients often need anti-allergic drugs to relieve asthma symptoms, and the dosage and type can be used as an indicator of asthma severity (46). The different rescue medications are scored on a scale of 1 to 3 as shown in Table 4, and the TAMS expressed as a sum of the individual scores.

\section{Lung function test}

Pulmonary function examination is an objective means of evaluating asthma control. Clinically, the main indicators 
Table 3 Symptom scores for asthma symptoms during daytime and nighttime

\begin{tabular}{lll}
\hline Score & Daytime symptom score & Nighttime symptom score \\
\hline 0 & No symptoms & No symptoms \\
1 & Symptoms are rare and short lasting & Wake up once or wake up early \\
2 & Two or more short lasting symptoms & Wake up twice, including wake up early \\
3 & Mild symptoms for more of the day, but had little impact on life and work & Wake up many times ( $\geq 3$ times) \\
4 & Severe symptoms for more of the day and affect life and work & Can't fall asleep at night \\
5 & The symptom is so serious that the subject cannot work and live normally & \\
\hline
\end{tabular}

Table 4 Total asthma medicine score (TAMS)

\begin{tabular}{ll}
\hline Score & Medication \\
\hline 1 & Oral and/or topical antihistamine \\
& Anti-leukotrienes \\
& Bronchodilator drugs, $\beta_{2}$ receptor agonists \\
& Bronchodilator drugs \\
& Nasal corticosteroids/inhaled corticosteroids \\
3 & Oral corticosteroids \\
& Combination therapy (corticosteroids and $\beta_{2}$ receptor agonists) \\
\hline
\end{tabular}

used for evaluating asthma pulmonary function are forced vital capacity (FVC), FEV1, and maximum PEF; all of which objectively reflect the severity of airway obstruction and therefore most commonly used indices of asthma severity (73).

\section{Asthma control test (ACT)}

ACT is an effective tool to assess the degree of asthma control for adults and adolescents above 12 years old. ACT score ranges from 5-25 points; with a score of 20-25 points = well controlled; $16-19$ points = not well controlled; and $5-15$ points $=$ poorly controlled (73). The childhood asthma control test (C-ACT) is for assessing asthma control in children aged 4-11 years old (74). C-ACT score ranges from 0 to 27 points; with a score of $20-27$ points $=$ well controlled, and 0-19 points $=$ not well controlled.

\section{Management of adverse reactions}

Adverse reactions can be divided into local and systemic types. A comprehensive review of studies investigating the efficacy of SLIT has indicated that the incidence of adverse reactions to SLIT in AR and/or AS was about $12.9 \%$, and that almost no patients required hospitalization or withdrew from the study because of AEs (75). Local reactions mainly include pruritus and swelling of mouth, tongue, eye, or lip, nose bleeding, headache, local skin rushes, aggravating rhinitis, and gastrointestinal effects (Table 5). The AEs can be early (<30 minutes) or delayed (>30 minutes) and graded to be Mild, Moderate, Severe, or of Unknown severity (76) (Table 5). The major systemic adverse reactions include asthma, urticaria, fever, and upper respiratory tract infection; however, importantly systemic reactions and anaphylactic reactions are extremely rare.

Although SLIT is a relatively safe and well-tolerated treatment option overall, it is worth noting that patients and guardians should nevertheless be instructed on how to identify AEs because SLIT is usually self-administered at home without direct medical supervision (27).

\section{Summary and future perspective}

China is an expansive country supporting nearly $20 \%$ of the world's population, with a large number of AR and AS patients. Presently only one SLIT product has been approved and used for HDM-induced AR and AS for more 
Table 5 Grading system for SLIT-associated local adverse events (AEs)

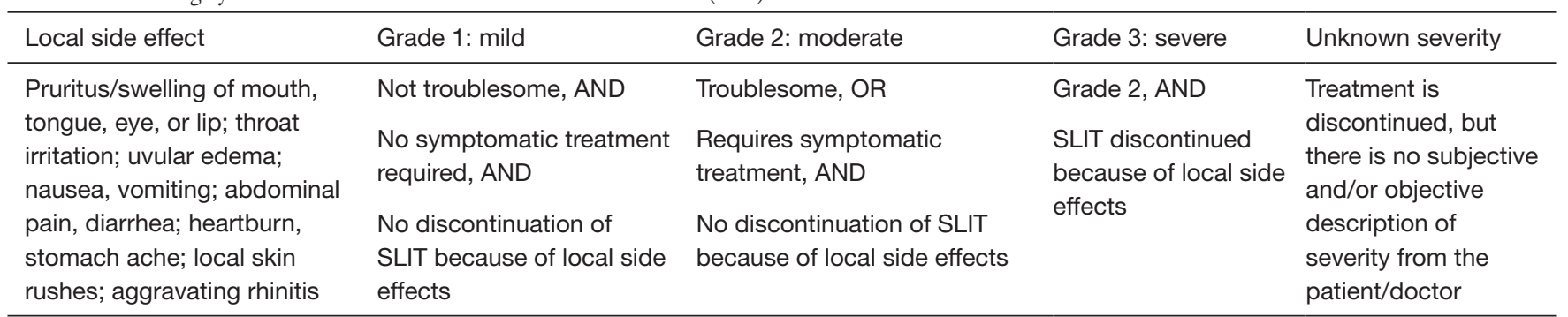

Each local AE can be early ( $<30$ minutes) or delayed. SLIT, sublingual immunotherapy.

than 10 years in China, and thus more SLIT products and clinical application experience for HDM are clearly needed. Indeed, as artemisia pollen allergy is a unique local issue affecting a large number of individuals especially in northern China, there is also an urgent need for immunotherapy products for treatment of artemisia pollen allergy in this vast area of China. Actually, this need is currently being addressed following registration of a clinical trial, which will investigate the efficacy and safety of a SLIT product for artemisia pollen allergy, and will hopefully be on the market in the near future. Although immunotherapy products for management of many of the clinically relevant allergens in China are still not available, more such products are currently being developed and expected to be commercialized in the future.

\section{Acknowledgments}

Funding: This work was supported by grants from the program for the Changjiang scholars and innovative research team (IRT13082) and the National Natural Science Foundation of China $(81630023,81725004)$.

\section{Footnote}

Conflicts of Interest: The authors have no conflicts of interest to declare.

Ethical Statement: The authors are accountable for all aspects of the work in ensuring that questions related to the accuracy or integrity of any part of the work are appropriately investigated and resolved.

\section{References}

1. Matricardi PM. 99th Dahlem conference on infection, inflammation and chronic inflammatory disorders: controversial aspects of the 'hygiene hypothesis'. Clin Exp Immunol 2010;160:98-105.

2. Wang XD, Zheng M, Lou HF, et al. An increased prevalence of self-reported allergic rhinitis in major Chinese cities from 2005 to 2011. Allergy 2016;71:1170-80.

3. Huang K, Yang T, Xu J, et al. Prevalence, risk factors, and management of asthma in China: a national cross-sectional study. Lancet 2019;394:407-18.

4. Zhao J, Bai J, Shen K, et al. Self-reported prevalence of childhood allergic diseases in three cities of China: a multicenter study. BMC Public Health 2010;10:551.

5. National Cooperative Group on Childhood Asthma; Institute of Environmental Health and Related Product Safety, Chinese Center for Disease Control and Prevention, et al. Third nationwide survey of childhood asthma in urban areas of China. Zhonghua Er Ke Za Zhi 2013;51:729.

6. Roberts G, Pfaar O, Akdis CA. EAACI guidelines on allergen immunotherapy: allergic rhinoconjunctivitis. Allergy 2018;73:765-98.

7. Saporta D. Sublingual immunotherapy: a useful tool for the allergist in private practice. Biomed Res Int 2016;2016:9323804.

8. Canonica GW, Cox L, Pawankar R, et al. Sublingual immunotherapy: World Allergy Organization position paper 2013 update. World Allergy Organ J 2014;7:6.

9. Bousquet J, Khaltaev N, Cruz AA, et al. Allergic Rhinitis and its Impact on Asthma (ARIA) 2008 update (in collaboration with the World Health Organization, GA(2) LEN and AllerGen). Allergy 2008;63 Suppl 86:8-160.

10. Zhang Y, Zhang L. Prevalence of allergic rhinitis in China. Allergy Asthma Immunol Res 2014;6:105-13.

11. Zhang $\mathrm{Y}$, Zhang L. Increasing prevalence of allergic rhinitis in China. Allergy Asthma Immunol Res 2019;11:156-69. 
12. Wang XY, Ma TT, Wang XY, et al. Prevalence of polleninduced allergic rhinitis with high pollen exposure in grasslands of northern China. Allergy 2018;73:1232-43.

13. Subspecialty Group of Rhinology, Editorial Board of Chinese Journal of Otorhinolaryngology Head and Neck Surgery; Subspecialty Group of Rhinology, Society of Otorhinolaryngology Head and Neck Surgery, Chinese Medical Association. Chinese guidelines for diagnosis and treatment of allergic rhinitis. Zhonghua Er Bi Yan Hou Tou Jing Wai Ke Za Zhi 2016;51:6-24.

14. Ellwood P, Asher MI, Beasley R, et al. The International Study of Asthma and Allergies in Childhood (ISAAC): phase three rationale and methods. Int J Tuberc Lung Dis 2005;9:10-6.

15. Kong WJ, Chen JJ, Zheng ZY, et al. Prevalence of allergic rhinitis in 3-6-year-old children in Wuhan of China. Clin Exp Allergy 2009;39:869-74.

16. Song $\mathrm{Y}$, Wang $M$, Xie J, et al. Prevalence of allergic rhinitis among elementary and middle school students in Changsha city and its impact on quality of life. J Laryngol Otol 2015;129:1108-14.

17. Zhang YM, Zhang J, Liu SL, et al. Prevalence and associated risk factors of allergic rhinitis in preschool children in Beijing. Laryngoscope 2013;123:28-35.

18. He S, Li YJ, Chen J. Clinical features of allergic rhinitis in children of Shanghai, China. Genet Mol Res 2016. doi: 10.4238/gmr.15028118.

19. Pawankar R, Canonica G, Holgate S, et al. World Allergy Organization (WAO) white book on allergy, Update. 2013.

20. Lin J, Wang W, Chen P, et al. Prevalence and risk factors of asthma in mainland China: The CARE study. Respir Med 2018;137:48-54.

21. Chen ZG, Li YT, Wang WH, et al. Distribution and determinants of Dermatophagoides mites sensitization of allergic rhinitis and allergic asthma in China. Int Arch Allergy Immunol 2019;180:17-27.

22. Li J, Sun B, Huang Y, et al. A multicentre study assessing the prevalence of sensitizations in patients with asthma and/or rhinitis in China. Allergy 2009;64:1083-92.

23. Liu X, Ng CL, Wang DY. The efficacy of sublingual immunotherapy for allergic diseases in Asia. Allergol Int 2018;67:309-19.

24. Baldo BA. Standardization of allergens. Examination of existing procedures and the likely impact of new techniques on the quality control of extracts. Allergy 1983;38:535-46.

25. Cao LF, Lu Q, Gu HL, et al. Clinical evaluation for sublingual immunotherapy of allergic asthma and atopic rhinitis with Dermatophagoides farinae drops. Zhonghua Er Ke Za Zhi 2007;45:736-41.

26. Li TY, Chen DH, Lin ZB, et al. Efficacy of sublingual immunotherapy with Dermatophagoides farinae drops in patients with allergic rhinitis. Zhonghua Er Bi Yan Hou Tou Jing Wai Ke Za Zhi 2011;46:859-62.

27. Cheng L, Zhou WC. Sublingual immunotherapy of house dust mite respiratory allergy in China. Allergol Immunopathol (Madr) 2019; 47:85-9.

28. Zhu L, Zhu LP, Chen RX, et al. Clinical efficacy of subcutaneous and sublingual immunotherapy in mitesensitized patients with allergic rhinitis. Zhonghua Er Bi Yan Hou Tou Jing Wai Ke Za Zhi 2011;46:986-91.

29. Wang DH, Chen L, Cheng L, et al. Fast onset of action of sublingual immunotherapy in house dust mite-induced allergic rhinitis: a multicenter, randomized, double-blind, placebo-controlled trial. Laryngoscope 2013;123:1334-40.

30. Cui L, Xia Z, Wang Z, et al. Efficacy of sublingual immunotherapy with Dermatophagoides farina drops in monosensitized and polysensitized patients with allergic rhinitis. Lin Chung Er Bi Yan Hou Tou Jing Wai Ke Za Zhi 2015;29:1094-7.

31. Lin Z, Liu Q, Li T, et al. The effects of house dust mite sublingual immunotherapy in patients with allergic rhinitis according to duration. Int Forum Allergy Rhinol 2016;6:82-7.

32. Meng Q, Liu X, Li P, et al. The influence of house dust mite sublingual immunotherapy on the TSLP-OX40L signaling pathway in patients with allergic rhinitis. Int Forum Allergy Rhinol 2016;6:862-70.

33. Lin $\mathrm{X}$, Lin $\mathrm{H}$, Wei $\mathrm{X}$, et al. The efficacy and safety of sublingual immunotherapy in children and adult patients with allergic rhinitis. Allergol Immunopathol (Madr) 2017;45:457-62.

34. Han L, You QJ, Li Z, et al. Evaluation of efficacy of 1 year after completing the 2-year specific sublingual immunotherapy treatment course in allergic rhinitis. Zhonghua Er Bi Yan Hou Tou Jing Wai Ke Za Zhi 2017;52:497.

35. Han M, Chen Y, Wang M. Sublingual immunotherapy for treating adult patients with allergic rhinitis induced by house dust mite among Chinese Han population. Medicine 2018;97:e11705.

36. Wen Y, Zhou L, Li Y, et al. Role of leptin in allergic rhinitis during sublingual immunotherapy. Eur Arch Otorhinolaryngol 2018;275:2733-8.

37. Wang Z, Li W, Chen $\mathrm{H}$, et al. Effect of sublingual immunotherapy on level of cytokines in PBMCs of patients 
with allergic asthma. J Huazhong Univ Sci Technolog Med Sci 2011;31:376-8.

38. Lin Z, Zhou L, Luo X, et al. Suppression of TIM-1 predicates clinical efficacy of sublingual immunotherapy for allergic rhinitis in children. Int J Pediatr Otorhinolaryngol 2013;77:1345-9.

39. Tian M, Wang Y, Lu Y, et al. Effects of sublingual immunotherapy for Dermatophagoides farinae on Th17 cells and CD4+ CD25+ regulatory T cells in peripheral blood of children with allergic asthma. Int Forum Allergy Rhinol 2014;4:371-5.

40. Shao J, Cui YX, Zheng YF, et al. Efficacy and safety of sublingual immunotherapy in children aged 3-13 years with allergic rhinitis. Am J Rhinol Allergy 2014;28:131-9.

41. Li P, Li Q, Huang Z, et al. Efficacy and safety of house dust mite sublingual immunotherapy in monosensitized and polysensitized children with respiratory allergic diseases. Int Forum Allergy Rhinol 2014;4:796-801.

42. Chen S, Zeng X, Wang L, et al. Effects of house dust mite sublingual immunotherapy in children with allergic rhinitis and asthma. Zhonghua Er Bi Yan Hou Tou Jing Wai Ke Za Zhi 2015;50:627-31.

43. Wang C, Wang K, Liu S, et al. Decreased level of osteopontin in children with allergic rhinitis during sublingual immunotherapy. Int J Pediatr Otorhinolaryngol 2016;81:15-20.

44. Yin GQ, Jiang WH, Wu PQ, et al. Clinical evaluation of sublingual administration of dust mite drops in the treatment of allergic asthma and allergic rhinitis of children. Eur Rev Med Pharmacol Sci 2016;20:4348-53.

45. Luo X, Hong $H$, Tang $J$, et al. Increased expression of miR-146a in children with allergic rhinitis after allergenspecific immunotherapy. Allergy Asthma Immunol Res 2016;8:132-40.

46. Zhong C, Yang W, Li Y, et al. Clinical evaluation for sublingual immunotherapy with Dermatophagoides farinae drops in adult patients with allergic asthma. Ir J Med Sci 2018;187:441-6.

47. Wang Y, Li C, Xu Y, et al. Sublingual immunotherapy decreases expression of interleukin-33 in children with allergic rhinitis. Indian J Pediatr 2018:85:872-6.

48. Tang LX, Yang XJ, Wang PP, et al. Efficacy and safety of sublingual immunotherapy with Dermatophagoides farinae drops in pre-school and school-age children with allergic rhinitis. Allergol Immunopathol (Madr) 2018;46:107-11.

49. Cui L, Li J, Li Y, et al. Long-term efficacy of sublingual mite immunotherapy in monosensitized and polysensitized children with allergic rhinitis: a 7-year prospective study.
Int Arch Allergy Immunol 2019;180:144-9.

50. Chen Z, Qian Y, Liu S, et al. Onset time and efficacy of sublingual immunotherapy with Dermatophagoides farinae drops in children with allergic rhinitis. Zhonghua Er Bi Yan Hou Tou Jing Wai Ke Za Zhi 2015;50:622-6.

51. Noon L. Prophylactic inoculation against hay fever. Lancet 1911:1572-3.

52. Tanaka Y, Nagashima H, Bando K, et al. Oral CD103$\mathrm{CD} 11 \mathrm{~b}+$ classical dendritic cells present sublingual antigen and induce Foxp3 + regulatory T cells in draining lymph nodes. Mucosal Immunol 2017;10:79-90.

53. Zhang YZ, Luo J, Wang ZH, et al. Efficacy and safety of sublingual dust mite drops in children with monoor polysensitized allergic rhinitis. Am J Otolaryngol 2019;40:755-60.

54. Bohle B, Kinaciyan T, Gerstmayr M, et al. Sublingual immunotherapy induces IL-10-producing T regulatory cells, allergen-specific T-cell tolerance, and immune deviation. J Allergy Clin Immunol 2007;120:707-13.

55. O'Hehir RE, Gardner LM, De Leon MP, et al. House dust mite sublingual immunotherapy - the role for TGF-beta and functional regulatory T cells. Am J Respir Crit Care Med 2009;180:936-47.

56. James LK, Shamji MH, Walker SM, et al. Long-term tolerance after allergen immunotherapy is accompanied by selective persistence of blocking antibodies. J Allergy Clin Immunol 2011;127:509-516.e1.

57. Gidaro GB, Marcucci F, Sensi L, et al. The safety of sublingual-swallow immunotherapy: an analysis of published studies. Clin Exp Allergy 2005;35:565-71.

58. Bernstein DI, Bardelas JA, Svanholm Fogh B, et al. A practical guide to the sublingual immunotherapy tablet adverse event profile: implications for clinical practice. Postgrad Med 2017;129:590.

59. Suárez-Fueyo A, Ramos T, Galán A, et al. Grass tablet sublingual immunotherapy downregulates the TH2 cytokine response followed by regulatory T-cell generation. J Allergy Clin Immunol 2014;13:130-8.e1-2.

60. Bao Y, Chen J, Cheng L, et al. Chinese guideline on allergen immunotherapy for allergic rhinitis. J Thorac Dis 2017;9:4607-50.

61. Wang T, Li Y, Wang F, et al. Nonadherence to sublingual immunotherapy in allergic rhinitis: a real-life analysis. Int Forum Allergy Rhinol 2017;7:389-92.

62. Chen J, Kong W, Xiang J, et al. Compliance analysis of sublingual immunotherapy and countermeasures. Lin Chuang Er Bi Yan Hou Tou Jing Wai Ke Za Zhi 2010;24:203-6. 
63. Vita D, Caminiti L, Ruggeri P, et al. Sublingual immunotherapy: adherence based on timing and monitoring control visits. Allergy 2010;65:668-9.

64. Xiang L, Zhao J, Bao YX, et al. Expert consensus on mite specific immunotherapy for airway allergic diseases in children. Chinese Journal of Applied Clinical Pediatrics 2018;3:1215-23.

65. Pitsios C, Demoly P, Bilò MB, et al. Clinical contraindications to allergen immunotherapy: an EAACI position paper. Allergy 2015;70:897-909.

66. Pfaar O, Lou H, Zhang Y, et al. Recent developments and highlights in allergen immunotherapy. Allergy 2018;73:2274-89.

67. Demoly P, Passalacqua G, Calderon MA, et al. Choosing the optimal dose in sublingual immunotherapy: Rationale for the 300 index of reactivity dose. Clin Transl Allergy 2015;5:44.

68. Mosbech H, Canonica GW, Backer V, et al. SQ house dust mite sublingually administered immunotherapy tablet (ALK) improves allergic rhinitis in patients with house dust mite allergic asthma and rhinitis symptoms. Ann Allergy Asthma Immunol 2015;114:134-40.

69. Canonica GW, Baena-Cagnani CE, Bousquet J, et al. Recommendations for standardization of clinical trials with allergen specific immunotherapy for respiratory allergy. A statement of a World Allergy Organization (WAO) taskforce. Allergy 2007;62:317-24.

70. Dietz de Loos DA, Segboer CL, Gevorgyan A, et al.

Cite this article as: $\mathrm{Li} \mathrm{H}$, Chen S, Cheng L, Guo Y, Lai H, Li Y, Lin X, Liu Z, Qiu Q, Shao J, Shi L, Tian M, Wang C, Wang H, Wang X, Wei Q, Wei Y, Xiang L, Yang Q, Zhao C, Zhang H, Zhi Y, Gao J, Li Q, Liu J, Wang K, Zhou W, Zhang L; Chinese Society of Allergy (CSA). Chinese guideline on sublingual immunotherapy for allergic rhinitis and asthma. J Thorac Dis 2019;11(12):4936-4950. doi: 10.21037/jtd.2019.12.37
Disease-specific quality-of-life questionnaires in rhinitis and rhinosinusitis: review and evaluation. Curr Allergy Asthma Rep 2013;13:162-70.

71. Pfaar O, Demoly P, Gerth van Wijk R, et al. Recommendations for the standardization of clinical outcomes used in allergen immunotherapy trials for allergic rhinoconjunctivitis: an EAACI Position Paper. Allergy 2014;69:854-67.

72. Gosepath J, Amedee RG, Mann WJ. Nasal provocation testing as an international standard for evaluation of allergic and nonallergic rhinitis. Laryngoscope 2005;115:512-6.

73. Global strategy for asthma management and prevention: update 2018. Global initiative for asthma. Available online: http://www.ginaasthma.org/

74. Liu AH, Zeiger RS, Sorkness CA, et al. The childhood asthma control test: retrospective determination and clinical validation of a cut point to identify children with very poorly controlled asthma. J Allergy Clin Immunol 2010;126:267-73, 273.e1.

75. Cox LS, Linnemann DL, Nolte H, et al. Sublingual immunotherapy: A comprehensive review. J Allergy Clin Immunol 2006;117:1021-35.

76. Passalacqua G, Baenacagnani CE, Bousquet J, et al. Grading local side effects of sublingual immunotherapy for respiratory allergy: speaking the same language. J Allergy Clin Immunol 2013;132:93-8. 